\title{
Effect of Uncomplicated Cataract Surgery on Central Macular Thickness in Diabetic and Non-diabetic Subjects
}

\author{
Brahm Prakash Guliani, MS Ophthal'; Isha Agarwal, MBBS ${ }^{1}$; Mayuresh P. Naik, MBBS, MS, DNB ${ }^{2}$ \\ ${ }^{1}$ Department of Ophthalmology, Vardhaman Mahavir Medical College \& Safdarjung Hospital, New Delhi, India \\ ${ }^{2}$ Department of Ophthalmology, Hamdard Institute of Medical Sciences \& Research, Hakeem Abdul Hameed Centenary \\ Hospital, New Delhi, India \\ ORCID: \\ Mayuresh Naik: https://orcid.org/0000-0002-7167-557X
}

\section{Abstract}

Purpose: To assess the quantitative changes of macula in diabetic and non-diabetic eyes after uncomplicated cataract surgery.

Methods: In this prospective interventional study being performed in a tertiary healthcare hospital, a total of 660 eyes were divided into two groups. Group 1 included 330 eyes from healthy subjects and group 2 included 330 eyes from well-controlled diabetic subjects with no diabetic retinopathy planned for phacoemulsification with foldable IOL implantation by the same surgeon under similar settings. Optical Coherence Tomography (Heidelberg Spectralis SD-OCT) was used to assess preoperative and postoperative central macular thickness (CMT) at weeks 1 and 6.

Results: The mean CMT in group 1 preoperatively, at postoperative week 1, and at post-operative week 6 was $257.03 \pm 20.904,262.82 \pm 17.010$, and $265.15 \pm 20.078 \mu \mathrm{m}$, respectively. The corresponding values in group 2 were $255.36 \pm 17.852,259.15 \pm 16.644$, and $266.09 \pm 18.844 \mu \mathrm{m}$, respectively. There was no significant difference in the mean CMT values between the two groups on any of the three occasions when the CMT was measured ( $P=0.374$ and $P=0.313$ at weeks 1 and 6 , respectively).

Conclusion: There was no statistically significant difference in CMT between normal subjects and diabetic subjects without diabetic retinopathy preoperatively and in early postoperative period after uncomplicated phacoemulsification surgery.

Keywords: Central Macular Thickness; Diabetic Macular Edema; Uncomplicated Phacoemulsification

J Ophthalmic Vis Res 2019; 14 (4): 442-447

\section{Correspondence to:}

Mayuresh P. Naik, MBBS, MS, DNB. Room No. 3 of Eye OPD, First floor of OPD building, Department of Ophthalmology, H.I.M.S.R \& H.A.H.C Hospital, near GK2, Alaknanda, New Delhi 110062, India.

E-mail: mayureshpnaik@gmail.com

Received: 17-02-2018Ａccepted: 24-03-2019

\section{Access this article online}

Website:

https://knepublishing.com/index.php/JOVR

DOI:

10.18502/jovr.v14i4.5447

\section{INTRODUCTION}

Cataract extraction is one of the most commonly performed ophthalmic surgeries. Recent innovations in instrumentation, lens design, and surgical technique have improved the outcome of cataract surgery. ${ }^{[1]}$ Currently, the preferred technique is

This is an open access journal, and articles are distributed under the terms of the Creative Commons Attribution-NonCommercial-ShareAlike 4.0 License, which allows others to remix, tweak, and build upon the work non-commercially, as long as appropriate credit is given and the new creations are licensed under the identical terms.

How to cite this article: Guliani BP, Agarwal I, Naik MP. Changes in CMT after Cataract Surgery. J Ophthalmic Vis Res 2019;14:442-447. 
phacoemulsification using small incisions and implantation of a foldable intraocular lens (IOL). ${ }^{[2]}$ This is an efficient procedure, and uneventful surgery is generally associated with good visual results. ${ }^{[3]}$ However, the development of cystoid macular edema (CME) can lead to suboptimal postoperative vision. ${ }^{[4]}$ This can occur in patients with ocular diseases, such as uveitis or diabetic retinopathy (DR), even after uncomplicated cataract surgery. ${ }^{[5]}$

CME following cataract surgery was initially reported by Irvine in 1953 and demonstrated angiographically by Gass and Norton in 1966 and has come to be known as the Irvine Gass syndrome. ${ }^{[6]}$

It is not uncommon to encounter CME in otherwise healthy eyes after uneventful phacoemulsification surgery. ${ }^{[7]}$ The incidence after phacoemulsification is reported to be $0.1-2 \%$ in healthy populations. ${ }^{[7]}$

Although the exact pathophysiology is not known, the role of surgical trauma with the release of prostaglandins and blood-retinal barrier disruption is suspected. ${ }^{[8]}$ Light toxicity and vitreomacular traction might also have a role. ${ }^{[9]}$

The incidence of pseudophakic CME depends on the methodology used in its detection. It has been suggested that prophylactic use of nonsteroidal anti-inflammatory drugs preoperatively, and steroids and anti-inflammatory drugs in the postoperative period, reduces the incidence of postoperative pseudophakic CME. ${ }^{[10]}$

Diabetes mellitus increases the probability of developing cataract and the risk of decreased visual outcomes after cataract surgery. ${ }^{[7]}$

It has been suggested that in diabetics macular edema after cataract surgery occurs predominantly in those with concurrent pre-existing diabetic macular edema (DME) involving the center of the macula. ${ }^{[11]}$ While others have reported that the development of postoperative macular edema does not need pre-existing DME, ${ }^{[1]}$ these were published before the availability of optical coherence tomography (OCT) technology. The dynamics of macular edema and cataract surgery in those with DR can be explored using the qualitative and quantitative OCT-based data.

Here we evaluate the retinal thickness changes in the early postoperative course of six weeks in non-diabetic and diabetic subjects after uncomplicated phacoemulsification with intracapsular IOL implantation.

\section{METHODS}

Ethical clearance was obtained from the Ethics Committee, the Institutional Review Board at Vardhaman Mahavir Medical College (V.M.M.C), and the Safdarjung Hospital, New Delhi.

This tertiary health center-based observational study was conducted on adult patients posted for cataract surgery at the Department of Ophthalmology, VMMC and Safdarjung Hospital, New Delhi. Assuming the effect size to be 0.8 (ratio of difference of two means/standard error), power to be $85 \%$, and the level of significance to be $5 \%$, a sample size of 330 eyes per group, including the $10 \%$ loss to follow-up, was required for the study, using software $\mathrm{G}$ power 3.1.

The patients were divided into two groups based on the inclusion and exclusion criteria as follows: GROUP 1, 330 healthy subjects planned for phacoemulsification with foldable IOL implantation by the same surgeon under similar settings; GROUP 2, 330 well-controlled diabetic subjects with no DR posted for phacoemulsification with foldable IOL implantation by the same surgeon under similar settings.

The inclusion criteria for group 1 (healthy subjects) included age $>40$ years and senile cataract undergoing uncomplicated cataract surgery. Patients with complicated cataract surgery, intraocular pressure $>21 \mathrm{mmHg}$, dense white cataract in whom OCT could not be performed, and any ocular diseases that might influence central macular thickness (CMT), such as glaucoma, uveitis, and age-related macular degeneration, were excluded from the study. Patients with a history of previous eye surgery or a history of macular edema in the fellow eye were also excluded.

Group 2 (well-controlled diabetic subjects) had similar inclusion criteria along with a diagnosis of diabetes mellitus of any duration, controlled on oral hypoglycemic agents or insulin, blood sugar $<200$ mg\% (All-India-Ophthalmological-Society (AIOS) Guidelines to Prevent Intraocular Infection, 2009) and $\mathrm{HbA} 1 \mathrm{C}<7 \%$ (American Diabetes Association ADA A1C Goals, Standards of Medical Care in Diabetes-2015), and an absence of any evidence of DR as assessed by indirect ophthalmoscopy and OCT. $^{[12]}$ Besides similar exclusion criteria as for group 1, group 2 patients were also excluded based on the presence of anemia [men $(>15 \mathrm{yr}),<$ 
$13 \mathrm{~g} / \mathrm{dL}$; women (> $15 \mathrm{yr}$ ), < $12 \mathrm{~g} / \mathrm{dL}],{ }^{[13]}$ pregnancy, or diabetic nephropathy (abnormal kidney function test including serum electrolytes, microalbuminuria, serum creatinine, and serum urea).

OCT (Heidelberg Spectralis SD-OCT) was used to assess pre- and postoperative (at weeks 1 and 6 ) CMT (central subfield thickness equating to mean thickness in the central 1000- $\mu \mathrm{m}$ diameter area).

Repeated measure analysis and multiple comparison correction with the Bonferroni method were applied to the data. $P<0.05$ was considered statistically significant.

\section{RESULTS}

The majority of the patients (47\%) were in the age group of 51-60 years [Table 1]. More than half of the patients in group 1 (54.5\%) and 39.4\% of patients in group 2 were between 51 and 60 years old (no significant difference when comparing groups, $P=$ 1.71).

The mean age in group 1 was $58.30 \pm 7.66$ years, while the mean age in group 2 was $63.24 \pm 9.74$ years $(P=2.89)$.

Overall, the study comprised of 390 (59\%) males and 270 (41\%) females [Table 1]. Group 1 comprised of $51.5 \%$ males and $48.5 \%$ females and group 2 of $66.7 \%$ males and $33.3 \%$ females $(P=2.12)$.

The mean CMT values in group 1 preoperatively and at postoperative weeks 1 and 6 were 257.03 \pm 20.90, $262.82 \pm 17.01$, and $265.15 \pm 20.07 \mu \mathrm{m}$, respectively [Table 2]. The mean CMT changes in group 1 preoperatively versus postoperative week 1 versus postoperative week 6 were statistically significant (both $P$-values $<0.001$ ). The mean CMT changes at postoperative weeks 1 and 6 were also significantly different $(P$-value $<0.001)$.

The mean CMT values in group 2 preoperatively, at postoperative week 1 , and at postoperative week 6 were $255.36 \pm 17.85,259.15 \pm 16.64$, and 266.09 $\pm 18.84 \mu \mathrm{m}$, respectively [Table 2].

The mean CMT changes in group 2 preoperatively versus postoperative week 1 versus postoperative week 6 were both statistically significant $(P$-value $<0.001)$. The mean CMT changes at postoperative weeks 1 and 6 were also significantly different ( $P$-value $<0.001)$.

No significant difference was noted in the mean CMT values between the two groups on any of the three occasions when CMT was measured [Table
2]. There was no significant change in the variation of mean CMT at weeks 1 and 6 postoperatively from baseline when groups 1 and 2 were compared [Table 2].

In our study, none of the patients developed clinical macular edema or CME on OCT.

\section{DISCUSSION}

This prospective comparative study was undertaken to assess the effect of uncomplicated phacoemulsification procedure with IOL implantation on CMT in diabetic and nondiabetic subjects in the early postoperative period (up to six weeks). The CMT used for comparison among the study subjects in our study corresponded to the mean thickness of all points in the central subfield of 1$\mathrm{mm}$ diameter of the ETDRS macular subfields. The CMT was assessed with OCT preoperatively, and at weeks 1 and 6 postoperatively, and comparisons were made between the measurements of the two study groups. The macula in healthy controls as well as in controlled diabetics without DR was increased significantly at the end of the first and sixth weeks postoperatively compared to the preoperative results. In both groups, this thickening persisted until six weeks postoperatively in all subjects and did not regress to preoperative levels till the last follow-up at six weeks. This study demonstrated that the influence of uncomplicated cataract surgery on CMT in well-controlled diabetic patients without DR did not significantly differ from healthy non-diabetic subjects after uncomplicated cataract surgery. In other words, well-controlled diabetics without DR and nondiabetic patients showed similar intragroup thickening of the central macular subfield at weeks 1 and 6 after uncomplicated phacoemulsification, and the intergroup comparison was not statistically significant.

The rate of development of macular edema following cataract surgery at different time intervals in people with diabetes (with or without $D R$ ) varies from $31 \%$ to $81 \% .{ }^{[14]}$ Certain minimal changes in the retina like subclinical CME and retinal leakage can occur even after uneventful cataract surgery. These subclinical changes in macular thickness after cataract surgery can be easily diagnosed on OCT and fluorescein angiography (FA). ${ }^{[15]}$ It has been reported by some studies that macular edema after cataract surgery, in people with diabetes, may occur predominantly in patients 
Table 1. Age- and sex-wise distribution of study subjects among the two study groups

\begin{tabular}{lcccc}
\hline Age Groups (Years) & \multicolumn{2}{c}{ GROUP 1 } & GROUP 2 \\
\hline $41-50$ & Male & Female & $20(6.06 \%)$ & $10(3.03 \%)$ \\
$51-60$ & $30(9.09 \%)$ & $20(6.06 \%)$ & $70(21.21 \%)$ & $60(18.18 \%)$ \\
$61-70$ & $100(30.30 \%)$ & $80(24.24 \%)$ & $50(15.15 \%)$ & $40(12.12 \%)$ \\
$>70$ & $40(12.12 \%)$ & $40(12.12 \%)$ & $40(12.12 \%)$ & $40(12.12 \%)$ \\
Total & $15(4.54 \%)$ & $5(1.51 \%)$ & $220(66.7 \%)$ & $110(33.3 \%)$ \\
Total & $170(51.5 \%)$ & $160(48.5 \%)$ & $330(100 \%)$ &
\end{tabular}

Table 2. Groups 1 and 2: Repeated measure analysis and multiple comparison correction with Bonferroni method depicting change in mean central macular thickness from baseline to postoperative week 1 and week 6

\begin{tabular}{|c|c|c|c|}
\hline & GROUP 1 & GROUP 2 & \\
\hline Pre-op CMT & $257.03 \pm 20.904$ & $255.36 \pm 17.852$ & \\
\hline Week 1 post-op CMT & $262.82 \pm 17.010$ & $259.15 \pm 16.644$ & \\
\hline Week 6 post-op CMT & $265.15 \pm 20.078$ & $266.09 \pm 18.844$ & \\
\hline $\begin{array}{l}\text { Change in mean CMT at } \\
\text { week } 1 \text { post-op as compared } \\
\text { to pre-op baseline }\end{array}$ & $\begin{array}{l}5.788 \pm 11.324(P \text {-value } \\
0.006 \text { for INTRAGROUP } \\
\text { COMPARISON) }\end{array}$ & $\begin{array}{l}3.788 \pm 6.066(P \text {-value } \\
0.001 \text { for INTRAGROUP } \\
\text { COMPARISON })\end{array}$ & $\begin{array}{l}P \text {-value } 0.374 \text { for } \\
\text { INTERGROUP COMPARISON }\end{array}$ \\
\hline $\begin{array}{l}\text { Change in mean CMT at } \\
\text { week } 6 \text { post-op as } \\
\text { compared to pre-op } \\
\text { baseline }\end{array}$ & $\begin{array}{l}8.121 \pm 11.056(P \text {-value }< \\
0.001 \text { for INTRAGROUP } \\
\text { COMPARISON })\end{array}$ & $\begin{array}{l}10.727 \pm 9.722(P \text {-value }< \\
\text { O.001 for INTRAGROUP } \\
\text { COMPARISON })\end{array}$ & $\begin{array}{l}P \text {-value } 0.313 \text { for } \\
\text { INTERGROUP COMPARISON }\end{array}$ \\
\hline $\begin{array}{l}\text { Change in mean CMT at } \\
\text { week } 6 \text { post-op as } \\
\text { compared to week } 1 \text { post-op }\end{array}$ & $\begin{array}{l}2.333 \pm 9.504(P \text {-value } \\
0.172 \text { for INTRAGROUP } \\
\text { COMPARISON })\end{array}$ & $\begin{array}{l}6.939 \pm 7.208(P \text {-value }< \\
\text { O.001 for INTRAGROUP } \\
\text { COMPARISON })\end{array}$ & $\begin{array}{l}P \text {-value } 0.336 \text { for } \\
\text { INTERGROUP COMPARISON }\end{array}$ \\
\hline
\end{tabular}

CMT, central macular thickness, pre-op, preoperative; post-op, postoperative

with concurrent pre-existing DME involving the center of the macula. On the other hand, some researchers have reported that for postoperative macular edema to develop, pre-existing DME is not required. ${ }^{[11]}$ However, these studies were completed prior to the availability of OCT technology. For detecting $\mathrm{CME}$, the sensitivity and specificity of OCT is $96 \%$ and $100 \%$, respectively, compared with FA. ${ }^{[16]}$ OCT can detect not only macular thickening before any angiographic evidence of macular edema but also produces reproducible and consistent quantitative results that are ideal for follow-up and assessment of the treatment response. ${ }^{[16,17]}$ For these reasons, we chose OCT as the investigative modality in our observational study.

There is some disagreement in the observations of various studies reporting an increase in CMT or development of macular edema after cataract surgery in patients with diabetes without DR. In a case-control study conducted on around 4,500 diabetics without preoperative macular edema, the incidence of postoperative macular edema was $4 \%$, which was higher than that in the population 
without diabetes $(P<0.001) \cdot{ }^{[17]}$ These authors also reported a higher risk for the development of macular edema (RR 1.80) in diabetic subjects without DR compared to patients without diabetes (RR 1.17). ${ }^{[17]}$ On the other hand, Katsimpris et al found increased macular thickness after uncomplicated cataract surgery in diabetics without DR compared to preoperative values or to a control group of patients at all follow-ups up to 12 months after cataract surgery. ${ }^{[18]}$ The eyes of diabetic patients without DR presented higher CMT and a higher incidence of CME after cataract surgery compared to the eyes of healthy controls, thus explaining the unsatisfactory visual acuity following cataract surgery in these patients. ${ }^{[18]}$ However, a recently conducted meta-analysis among diabetic patients without DR observed no statistically significant increase in CMT values after cataract surgery at one, three, and six months after cataract extraction. ${ }^{[19]}$

Many studies have postulated an association between progression of DR and cataract surgery, ${ }^{[20]}$ whereas other studies did not observe any significant association and consider any diabetic retinal changes as part of the natural course of the disease. ${ }^{[21]}$

In our study, the preoperative CMT measured by OCT is the same between the two groups. This is in accordance with a study conducted by Massin et al, who also found no differences in macular thickness comparing healthy subjects and diabetics without CME. ${ }^{[22]}$

There are, unfortunately, three limitations in our study. First, there are certain variables affecting the quality of OCT, despite it being a fast, noninvasive, non-contact, reproducible, and reliable invivo imaging technique. ${ }^{[23]}$ When media opacities, such as cataract, are present (especially in the form of cortical and subcapsular types), reliable scans might not be obtained preoperatively. ${ }^{[23]}$ To avoid this difficulty, we excluded patients with dense media opacities. Second, though our study showed no significant statistical difference between the two groups, few other studies have shown a significant rise in the CMT postoperatively in well-controlled diabetics with no DR. Therefore, large-scale studies with a longer followup period are likely required to accurately elucidate the role of diabetes control and DR status on the postoperative visual prognosis of patients undergoing uncomplicated phacoemulsification.
Last, the current study is limited by the duration of follow-up of patients that precludes any firm clinical conclusions based on the results of the study.

In conclusion, CMT is increased after uncomplicated phacoemulsification both at weeks 1 and 6 postoperatively in both healthy nondiabetic subjects and in well-controlled diabetic patients without DR; the difference between the two groups is not statistically significant. It is postulated that good diabetes control is needed to prevent an increase in CMT and postoperative macular edema after uncomplicated uneventful phacoemulsification procedure. However, long term follow-up studies may be required so that management algorithms can be formulated in order to dictate our surgical paradigms.

\section{Financial Support and Sponsorship}

Nil.

\section{Conflicts of Interest}

There are no conflicts of interest.

\section{REFERENCES}

1. DeCroos FC, Afshari NA. Perioperative antibiotics and anti-inflammatory agents in cataract surgery. Curr Opin Ophthalmol 2008;19:22-26.

2. Panchapakesan J, Rochtchina E, Mitchell P. Five-year change in visual acuity following cataract surgery in an older community: the Blue Mountains Eye Study. Eye 2004;18:278-282.

3. Linebarger EJ, Hardten DR, Shah GK, Lindstrom RL. Phacoemulsification and modern cataract surgery. Surv Ophthalmol 1999;44:123-147.

4. O'Brien TP. Emerging guidelines for use of NSAID therapy to optimize cataract surgery patient care. Curr Med Res Opin 2005;21:1131-1137. Review Erratum in: Curr Med Res Opin 2005;21:1431-1432.

5. Nelson ML, Martidis A. Managing cystoid macular edema after cataract surgery. Curr Opin Ophthalmol 2003;14:3943.

6. Nelson ML, Martidis A. Managing cystoid macular edema after cataract surgery. Curr Opin Ophthalmol 2003;14:3943.

7. Gass JD, Norton EW. Cystoid macular edema and papilledema following cataract extraction. A fluorescein fundoscopic and angiographic study. Arch Ophthalmol 1966;76:646-661. 
8. Ursell PG, Spalton DJ, Whitcup SM, Nussenblatt RB. Cystoids macular edema after phacoemulsification: relationship to blood-aqueous barrier damage and visual acuity. J Catarct Refract Surg 1999;25:1492-1497.

9. Flach AJ. The incidence, pathogenesis and treatment of cystoids macular edema following cataract surgery. Trans Am Ophthalmol Soc 1998;96:557-634.

10. Rho DS. Treatment of acute pseudophakic cystoid macular edema: diclofenac versus ketorolac. J Cataract Refract Surg 2003;29:2378-2384.

11. Kim SJ, Equi R, Bressler NM. Analysis of macular edema after cataract surgery in patients with diabetes using optical coherence tomography. Ophthalmology 2007;114:881889.

12. AIOS guidelines to prevent intraocular infection. 2009. Available from http://www.aios.org/guidelines endoph .pdf

13. FAO; WHO. World declaration and plan of action for nutrition. In: International Conference on Nutrition. Rome: Food and Agriculture Organization of the United Nations; December 1992.

14. Dowler JG, Sehmi KS, Hykin PG, Hamilton AM. The natural history of macular edema after cataract surgery in diabetes. Ophthalmology 1999;106:663-668.

15. Lara SA, Cakiner ET. Diabetes and cataract surgery: preoperative risk factors and positive nursing interventions. Insight 2014;39:18-20.

16. Biro Z, Balla Z, Kovacs B. Change of foveal and perifoveal thickness measured by OCT after phacoemulsification and
IOL implantation. Eye 2008;22:8-12.

17. Sahin M, Cingu AK, Gozum N. Evaluation of cystoid macular edema using optical coherence tomography and fundus autofluorescence after uncomplicated phacoemulsification surgery. J Ophthalmol 2013;2013:376013.

18. Katsimpris JM. , Petropoulos IK. , Zoukas G. , Patokos T. , Brinkmann C.K. , Theoulakis P.E. Central foveal thickness before and after cataract surgery in normal and in diabetic patients without retinopathy. Klin Monbl Augenheilkd 2012; 229:331-337.

19. Akcay BI, Bozkurt TK, Güney E, Unlü C, Erdogan G, Akcali G, et al. Quantitative analysis of macular thickness following uneventful and complicated cataract surgery. Clin Ophthalmol 2012;6:1507-1511.

20. Chu CJ, Johnston RL, Buscombe C, Sallam AB, Mohamed $Q$, Yang YC. Risk factors and incidence of macular edema after cataract surgery a database study of 81984 eyes. Ophthalmology 2016;123:316-323.

21. Liu J, Jones RE, Zhao J, Zhang J, Zhang F. Influence of uncomplicated phacoemulsification on central macular thickness in diabetic patients: a meta-analysis. PLOS ONE 2015;10:e0126343.

22. Massin P, Bandello F, Garweg JG, Hansen LL, Harding SP, Larsen $M$, et al. Safety and efficacy of ranibizumab in diabetic macular edema (RESOLVE study): A 12-month, randomized, controlled, double-masked, multicenter phase II study. Diabetes Care 2010;33:2399-405.

23. Henricsson M, Heijl A, Janzon L. Diabetic retinopathy before and after cataract surgery. $\mathrm{Br} J$ Ophthalmol 1996;80:789-793. 